\title{
Ideal glass transitions in thin films: An energy landscape perspective
}

\author{
Thomas M. Truskett* \& Venkat Ganesan \\ Department of Chemical Engineering and Institute for Theoretical Chemistry, \\ The University of Texas at Austin, Austin, TX 78712.
}

\begin{abstract}
We introduce a mean-field model for the potential energy landscape of a thin fluid film confined between parallel substrates. The model predicts how the number of accessible basins on the energy landscape and, consequently, the film's ideal glass transition temperature depend on bulk pressure, film thickness, and the strength of the fluid-fluid and fluid-substrate interactions. The predictions are in qualitative agreement with the experimental trends for the kinetic glass transition temperature of thin films, suggesting the utility of landscape-based approaches for studying the behavior of confined fluids.
\end{abstract}


Amorphous materials confined to small dimensions play a vital role in science and technology. Examples include biological fluids in membranes, oil trapped in porous rocks, lubricants, layered composites, and thin resist films used in the fabrication of microelectronic devices. Many of these systems exhibit large surface-to-volume ratios, and thus their physico-chemical properties are influenced by boundary and finite-size effects. ${ }^{1}$ The thermodynamic manifestations of confinement are diverse, ranging from shifts of the bulk phase boundaries to the creation of new phase transitions. ${ }^{2}$ Dynamics in thin films can also differ markedly from the corresponding bulk materials. Most notably, the glass transition can shift to either higher or lower temperatures upon confinement, depending on the nature of the interactions of the fluid and the confining medium. ${ }^{3-7}$

The ability to alter the physical properties of thin films by tuning film-substrate interactions represents a tremendous opportunity for the design and fabrication of advanced materials. However, progress hinges on understanding processes that occur at microscopic and/or mesoscopic length scales. Although theoretical and computational studies continue to provide fundamental insights, many questions concerning the molecular origins of thin-film phenomena remain unresolved. ${ }^{8-13}$ Hence, the development of a consistent and quantitative framework for modeling thin films is one of the outstanding challenges in the engineering and physical sciences.

In this Communication, we introduce and probe the utility of an "energy-landscape" based approach ${ }^{14}$ for describing the properties of thin films. Specifically, we develop a simple model for the topographical features of the energy landscape of a film confined between parallel substrates. We use this model to explore how the thermodynamic ideal glass (IG) transition temperature of the film (i.e., the temperature at which its configurational entropy vanishes) depends on bulk pressure, film thickness, and the strength of the fluid-fluid and fluid-substrate interactions. While the existence of an IG transition that underlies the laboratory glass transition of real materials remains an open and debated issue, it is a concept that has proven to be empirically useful and has strongly influenced modern thought on the glassy state. ${ }^{13}$ We find that the predicted IG transition of our model qualitatively reproduces experimental trends for the kinetic glass transition of thin films. 


\section{FLUID FILM MODEL}

The model that we consider is the soft-sphere/mean-field (SSMF) fluid. Its potential energy exhibits simple scaling properties, and thus it has served as the focus of several recent studies (albeit, in bulk homogeneous conditions). ${ }^{15,16}$ This fluid consists of $N$ sphericallysymmetric particles that interact via a soft-sphere repulsive pair potential $\epsilon\left(\sigma_{S S} / r\right)^{n}$ in addition to attractions quantified by a density-dependent mean-field form $-a_{b} \rho(\rho=N / V$ is the number density). The parameter $n$ determines the steepness of the soft-sphere repulsion, and it is typically taken to be in the range $8<n<16$. In the bulk situation, the potential energy per particle $\varphi\left(\mathbf{s}^{N}, \rho\right)$ can be expressed in terms of the scaled coordinates of the particles $\mathbf{s}_{i}=V^{-1 / 3} \mathbf{r}_{i}(i=1 \cdots N)$ as

$$
\varphi\left(\mathbf{s}^{N}, \rho\right)=-a_{b} \rho+y\left(\mathbf{s}^{N}\right) \eta_{b}^{n / 3}
$$

where $\eta_{b}=\pi \sigma_{S S}^{3} \rho / 6$ is the effective packing fraction of the molecules, and $y\left(\mathbf{s}^{N}\right)$ in Eq. (II) has the dimensions of energy per particle and is defined as

$$
y\left(\mathbf{s}^{N}\right) \equiv \frac{\epsilon}{N}\left(\frac{6}{\pi N}\right)^{n / 3} \times \sum_{i=1}^{N-1} \sum_{j=i+1}^{N} s_{i j}^{-n}
$$

with $s_{i j}=\left|\mathbf{s}_{\mathbf{i}}-\mathbf{s}_{\mathbf{j}}\right|$. In the situation where two parallel substrates (each with surface area $A$ ) confine the particle centers to a film of volume $V=A L$, both the packing fraction $\eta$ and the strength of the fluid-fluid attraction parameter $a$ are functions of the film thickness $L$, and the above model is modified as

$$
\varphi\left(\mathbf{s}^{N}, \epsilon_{L}, \rho\right)=-a \rho-\Psi+y\left(\mathbf{s}^{N}\right) \eta^{n / 3}
$$

where $\epsilon_{L}=\sigma_{S S} / L$ is the dimensionless reciprocal film thickness. Here, $-\Psi$ represents the attraction between the fluid particles and the confining substrates, incorporated in a mean-field manner. Expressions for $a, \Psi$, and $\eta$ have been derived elsewhere ${ }^{17-19}$ and are given by

$$
\begin{aligned}
& a=a_{b}\left[1-\frac{3}{4} \epsilon_{L}+\frac{1}{8} \epsilon_{L}^{3}\right] \\
& \Psi=\Psi_{0}\left[\epsilon_{L}-\frac{\epsilon_{L}^{3}}{\left(1+\epsilon_{L}\right)^{2}}\right] \\
& \eta=\frac{\pi \sigma_{S S}^{3} \rho}{6}\left[1-\frac{3}{16} \epsilon_{L}\right]
\end{aligned}
$$

The parameter $\Psi_{0}$ establishes the energy scale for the fluid-substrate interactions, and its connection to molecular parameters has been discussed previously. ${ }^{17,18}$ 


\section{POTENTIAL ENERGY LANDSCAPES OF THIN FILMS}

For given values of $\rho$ and $\epsilon_{L}, \varphi$ can be represented as a hypersurface in a $3 N+1$ dimensional space - the film's potential energy landscape. Despite the multidimensional nature of a material's energy landscape, only a few generic features of its "rugged" topography have been speculated to influence the thermodynamics and dynamics of fluids. ${ }^{14-16}$ Here, we develop a simple strategy to account for how confinement can impact these features. Since our primary focus is understanding the behavior of amorphous films, we restrict our attention to particle configurations devoid of crystalline order.

(i) Basin enumeration function $\sigma$ : This function quantifies the number of distinguishable minima on the landscape (inherent structures). Explicitly, if the total number of (amorphous) inherent structures on the landscape with well depths between $\phi$ and $\phi+d \phi$ is denoted $d \Omega$, then $\sigma\left(\phi, \epsilon_{L}, \rho\right)$ is given by the relationship $d \Omega=C \exp \left[N \sigma\left(\phi, \epsilon_{L}, \rho\right)\right] d \phi$, where $C$ is a scale factor with dimension reciprocal energy. One of the most simple and commonly used approximations for the distribution of inherent structure depths in bulk fluids is the Gaussian function. ${ }^{16}$ For the case of the SSMF fluid film, we propose the following simple generalization:

$$
\frac{\sigma\left(\phi, \epsilon_{L}, \rho\right)}{\sigma_{\infty}}=1-\left[\frac{\phi-\phi_{\infty}}{\phi_{\infty}-\phi_{m}}\right]^{2}, \phi_{m}<\phi<\phi_{\infty}
$$

where $\phi_{m} \equiv \phi_{m}\left(\epsilon_{L}, \rho\right)$ and $\phi_{\infty} \equiv \phi_{\infty}\left(\epsilon_{L}, \rho\right)$ are the potential energy at the minimum and maximum values of the basin enumeration function, respectively. The above expression retains the functional form proposed for bulk fluids, while rendering the quantities $\phi_{\infty}$ and $\phi_{m}$ film thickness $\left(\epsilon_{L}\right)$ dependent. Unfortunately, the accuracy of this approximation is unclear at present because the landscape statistics of thin films have not been systematically investigated by molecular simulation. Nonetheless, we take (5) as a workable starting point, and we leave exploration of more accurate approximations for future studies.

(ii) Mean inherent structure energy $\phi^{*}$ : At a given (reciprocal) temperature $\beta=1 / k_{B} T$, the fluid film will spend an overwhelming majority of its time in basins of attraction with inherent structures of energy $\phi^{*}=\phi^{*}\left(\epsilon_{L}, \rho, \beta\right)$, which satisfies ${ }^{15,16}$

$$
\left(\frac{\partial \sigma}{\partial \phi}\right)_{\epsilon_{L}, \rho}\left[\phi=\phi^{*}, \epsilon_{L}, \rho\right]=\beta
$$

Eq. (6) is exact ${ }^{15,16}$ if the intra-basin vibrational contribution to the free energy $f_{v i b}$ (see 
below) is independent of basin depth $\phi$. For the Gaussian landscape (5), we have

$$
\phi^{*}\left(\epsilon_{L}, \rho, \beta\right)= \begin{cases}\phi_{\infty}-\beta\left[\phi_{\infty}-\phi_{m}\right]^{2} / 2 \sigma_{\infty} & \beta<\beta^{I G} \\ \phi_{m} & \beta \geq \beta^{I G}\end{cases}
$$

where $\beta^{I G}=\beta^{I G}\left(\epsilon_{L}, \rho\right)$ locates the IG transition.

(iii) Configurational entropy $s_{C}$ : This quantity is defined as $s_{C} \equiv k_{B} \sigma\left(\phi^{*}, \epsilon_{L}, \rho\right)$ and thus is given by

$$
\frac{s_{C}\left(\epsilon_{L}, \rho, \beta\right)}{k_{B} \sigma_{\infty}}= \begin{cases}1-\left[\beta\left(\phi_{\infty}-\phi_{m}\right) / 2 \sigma_{\infty}\right]^{2} & \beta<\beta^{I G} \\ 0 & \beta \geq \beta^{I G}\end{cases}
$$

(iv) Ideal glass (IG) transition locus $\beta^{I G}$ : At temperatures below the IG transition, the configurational entropy of the film vanishes $s_{C}=0$, and the system is trapped in the amorphous basin with the lowest energy $\phi^{*}=\phi_{m}$ :

$$
\beta^{I G}\left(\epsilon_{L}, \rho\right)=\frac{2 \sigma_{\infty}}{\phi_{\infty}-\phi_{m}}
$$

(v) Helmholtz free energy $f$ : In terms of the above quantities, the film's Helmholtz free energy possesses a simple form: ${ }^{14-16}$

$$
f\left(\epsilon_{L}, \rho, \beta\right)=\phi^{*}-s_{C} / \beta k_{B}+f_{v i b}
$$

\section{IDEAL GLASS TRANSITION OF THE SSMF FLUID FILM}

Our development in the previous section briefly generalized the energy landscape formalism for bulk fluids ${ }^{14-16}$ to describe fluid films. Now we quote the explicit form of the above functions for the SSMF fluid film.

Using Eq. (3), (41), and (17)-(10), we obtain

$$
\beta^{I G}\left(\epsilon_{L}, \rho\right)=\frac{2 \sigma_{\infty}}{\left[y_{\infty}-y_{m}\right] \eta^{n / 3}}
$$

and

$$
\begin{aligned}
f\left(\epsilon_{L}, \rho, \beta\right) & =K(\beta)+y_{\infty} \eta^{n / 3}-\frac{\left[y_{\infty}-y_{m}\right]^{2}}{4 \sigma_{\infty}} \beta \eta^{2 n / 3} \\
& +\frac{n+2}{2 \beta} \ln \eta-a \rho-\Psi
\end{aligned}
$$


where $y_{m}$ and $y_{\infty}$ represent $y\left(\mathbf{s}^{N}\right)$ of Eq. (2) evaluated at $\phi_{m}$ and $\phi_{\infty}$, respectively. The term $K(\beta)$ depends only on temperature and does not enter into our present analysis. Following Shell et al., ${ }^{16}$ we have modeled the vibrational contribution to the free energy of Eq. (12) in the classical harmonic approximation. ${ }^{20}$ Other thermodynamic quantities of interest, like the transverse component of the pressure tensor $P_{\|}\left(\epsilon_{L}, \rho, \beta\right)=\rho(\partial f / \partial \ln \rho)_{\epsilon_{L}, \beta}$ and the chemical potential $\mu\left(\epsilon_{L}, \rho, \beta\right)=f+P_{\|} \rho^{-1}$ of the film, follow from Eq. (12).

To examine the confinement-induced shift of the IG transition for the SSMF fluid film, we consider the situation where the film is in equilibrium with a bulk fluid at pressure $P_{b}$; hence the "shift" we refer to is measured relative to the bulk IG transition at that pressure. We have determined the IG transition of the film numerically as well as by an approximate analytical theory. In both approaches, the IG transition of the bulk fluid is first determined by the condition $\beta_{b}^{I G}\left(P_{b}\right)=\beta^{I G}\left[\epsilon_{L}=0, \rho_{b}^{I G}\left(P_{b}\right)\right]$ (see Eq. (11)), where the bulk density $\rho_{b}^{I G}\left(P_{b}\right)=\rho_{b}\left(P_{b}, \beta_{b}^{I G}\right)$ follows from an inversion of the equation of state $P_{b}=P_{\|}\left(\epsilon_{L}=\right.$ $\left.0, \rho_{b}, \beta_{b}^{I G}\right)$. Similarly, the IG transition of the thin film $\beta^{I G}\left(\epsilon_{L}, P_{b}\right)=\beta^{I G}\left[\epsilon_{L}, \rho^{I G}\left(\epsilon_{L}, P_{b}\right)\right]$ is given by Eq. (11), where the density of the film at its IG transition is denoted $\rho^{I G}\left(\epsilon_{L}, P_{b}\right) \equiv$ $\rho\left(\epsilon_{L}, P_{b}, \beta^{I G}\right)$ and is determined by the condition that the film and the bulk fluid have equal chemical potentials; i.e. $\mu\left[\epsilon_{L}=0, \rho_{b}\left(P_{b}, \beta^{I G}\right), \beta^{I G}\right]=\mu\left[\epsilon_{L}, \rho, \beta^{I G}\right]$. Numerical calculations require values for the parameters $n, \sigma_{\infty}, y_{\infty}, y_{m}, a_{b}$, and $\Psi_{0}$. Here, we use $n=12, \sigma_{\infty}=$ $0.5368, y_{\infty}=61.73 \epsilon, y_{m}=53.22 \epsilon$, and $a_{b}=16.5 \epsilon \sigma^{3}$ (we examine several values of the fluidsubstrate attraction parameter $\Psi_{0}$ ). This set of parameters was chosen because it provides good qualitative agreement with both the liquid-state thermodynamics ${ }^{16}$ and the predicted IG transition locus ${ }^{21}$ of the bulk Lennard-Jones system.

A perturbation approach allows us to derive the shifts in the IG transition temperature $\Theta \equiv\left[\beta_{b}^{I G}\left(P_{b}\right)-\beta^{I G}\left(\epsilon_{L}, P_{b}\right)\right] / \beta^{I G}\left(\epsilon_{L}, P_{b}\right)$ to the first order in $\epsilon_{L}$. To maintain brevity, we avoid elaborating the algebraic details that accompany these calculations, and instead quote the following three equivalent results:

$$
\begin{aligned}
\Theta & \approx \frac{1}{\Delta c_{P, b}^{I G}}\left[-\left(\frac{\partial s_{C}}{\partial \epsilon_{L}}\right)_{P_{b}}\right]_{\epsilon_{L}=0} \epsilon_{L} \\
\Theta & \approx \frac{2 \Gamma_{b}^{I G} /\left\{\sigma_{S S} \rho_{b}^{I G}\right\}-3 / 16}{\kappa_{T, b}^{I G}}\left(-\frac{d \ln \beta_{b}^{I G}}{d P_{b}}\right) \epsilon_{L} \\
\Theta & \approx\left(\Psi_{0}-\frac{9 a_{b} \rho_{b}^{I G}}{8}\right) \rho_{b}^{I G}\left(-\frac{d \ln \beta_{b}^{I G}}{d P_{b}}\right) \epsilon_{L}
\end{aligned}
$$


Here, $\Delta c_{P, b}^{I G}\left(P_{b}\right)=-\left(\partial s_{C} / \partial \ln \beta\right)_{P_{b}}$ is the configurational heat capacity, $\kappa_{T, b}^{I G}\left(P_{b}\right)$ is the isothermal compressibility, $2 \Gamma_{b}^{I G}\left(P_{b}\right) / \sigma_{S S}=\left[\rho^{I G}\left(\epsilon_{L}, P_{b}\right)-\rho_{b}\left(P_{b}, \beta^{I G}\right)\right] / \epsilon_{L}$ is the surface excess density, and $\eta_{b}^{I G}\left(P_{b}\right)$ is the packing fraction, each evaluated at the IG transition of the bulk fluid, i.e. $\left[\epsilon_{L}=0, P_{b}, \beta_{b}^{I G}\left(P_{b}\right)\right]$. The thermodynamic quantities in (13) can be expressed ${ }^{22}$ as analytical functions of the molecular parameters of the model. Figure 1 displays the confinement-induced shifts in the IG transition temperature (whose physical implications are discussed below), demonstrating the excellent accuracy of the perturbation approach in capturing the full numerical results.

The above expressions (13) shed light on the physics of confinement-induced changes in the IG transition temperature. Consider the first equality of Eq. (13). Since $\Delta c_{P, b}^{I G}\left(P_{b}\right)>0$, this relation reveals the following simple rule for the (small $\epsilon_{L}$ ) shift of the IG transition. If confinement increases the number of basins on the landscape that the fluid can sample (hence increasing $s_{C}$ ), then the IG transition temperature is depressed. Conversely, if confinement decreases the number of accessible basins, then the IG transition temperature is elevated. This result does not depend upon a specific model for the film's energy landscape.

The second equality of Eq. (13) uses our model to connect the landscape-based perspective to physical quantities. Since $d \ln \beta^{I G} / d P_{b}<0$ for this model, it predicts that the direction of the shift of the IG transition is determined by the sign and magnitude of the film's surface excess density $2 \Gamma_{b}^{I G}\left(P_{b}\right)$. Large positive values of the surface excess density reduce the configurational entropy of the film and increase the IG transition temperature, while small or negative values of the surface excess have the opposite effect. While this result depends on our model for the energy landscape, we note that it is consistent with the recent theoretical predictions of McCoy and Curro. ${ }^{12}$ The reason that these two different approaches arrive at similar conclusions is easy to understand. McCoy and Curro's model begins with the hypothesis that the confinement-induced shift in the kinetic glass transition is determined by how confinement affects the density of the fluid film. Similarly, as can be seen by Eq. (11), the shift in the IG transition of our mean-field model is determined by how confinement affects the packing fraction of the molecules in the film.

The third equality of Eq. (13) establishes a molecular connection: strongly attracting walls $\Psi_{0} \sigma_{S S}^{3} / a_{b}>>1$ elevate the IG transition while neutral or repulsive walls $\Psi_{0} \sigma_{S S}^{3} / a_{b} \approx 0$ depress the IG transition. Fig. 1 shows the confinement-induced shifts in the IG transition temperature as predicted by the linear expression of Eq. (13) and the full non-linear model of 
Eq. (11) and (12). As can be seen, the shift of the IG transition is approximately inversely proportional to film thickness down to molecular length scales. As has been discussed extensively elsewhere, these trends are in good qualitative agreement with the experimental shifts in the glass transition temperature of confined fluids. ${ }^{3,4,6}$

Finally, we note that the glass transition shifts shown in Fig. 1 correspond to a fixed value of the bulk pressure $P_{b}=0$. It is straightforward to employ the model and the framework outlined above to analyze the effects of varying the bulk pressure. Preliminary calculations indicate that while qualitatively similar results are seen at higher pressures, reducing the pressure (placing the fluid under tension) can change both the sign and the magnitude of shifts. $^{22}$ A comprehensive investigation of pressure effects on the glass transition is beyond the scope of this Communication and is deferred to a future publication.

\section{CONCLUSIONS}

We have examined the confinement-induced changes in the energy landscape of a simple statistical mechanical model of a fluid film. Our analysis here focuses on elucidating how the film's IG transition depends on various molecular and macroscopic parameters. The model predictions are qualitatively consistent with theoretical and experimental studies, thereby suggesting that landscape based approaches may serve to provide a framework for predicting the thermodynamic and dynamic properties of confined fluids. It is appropriate to point out that the SSMF film model, although conceptually useful, should only be viewed as a starting point that suggests the viability of the inherent structure formalism ${ }^{14}$ for understanding the behavior of confined fluids and thin films. In fact, many films of scientific interest exhibit either narrow confining geometries and/or strong, directional fluid-substrate attractions that are not amenable to a mean-field treatment or the Gaussian landscape approximation. In such cases, molecular simulations can be employed to extract information about how confinement alters their energy landscapes, and hence their physico-chemical properties. We are currently pursuing research along these lines.

We thank Scott Shell, Pablo Debenedetti, Emilia La Nave and Francesco Sciortino for sending us a preprint of their recent manuscript ${ }^{16}$, which played an important role in motivating the present work. VG gratefully acknowledges support from National Science Foundation under Award Number DMR-02-04199, and the Petroleum Research Fund, administered by 
the ACS.

\section{REFERENCES}

*Author to whom correspondence should be addressed.

${ }^{1}$ J. M. Drake and J. Klafter, Phys. Today 43, 46-55 (1990).

${ }^{2}$ L. D. Gelb, K. E. Gubbins, R. Radhakrishnan, and M. Sliwinska-Bartkowiak, Rep. Prog. Phys. 62, 1573 (1999);

${ }^{3}$ J. A. Forrest and K. Dalnoki-Veress, Adv. Colloid Interface Sci. 94, 167 (2001).

${ }^{4}$ G.B. McKenna, Journal de Physique IV (France) 10, Pr7-53 (2000).

${ }^{5}$ J. Schüller, R. Richert, and E. W. Fischer, Phys. Rev. B 52, 15232 (1995).

${ }^{6}$ D. S. Fryer, P. F. Nealey, J. J. de Pablo, Macromolecules 33, 3376 (2000); D. S. Fryer et al., Macromolecules 34, 5627 (2001); R. S. Tate et. al., J. Chem. Phys. 115, 9982 (2001).

${ }^{7}$ R. A. L. Jones, Curr. Opin. Colloid Interface Sci. 4, 153 (1999).

${ }^{8}$ C. Mischler, J. Baschnagel, and K. Binder, Adv. Colloid Interface Sci. 94, 197 (2001).

9 J. A. Torres, P. F. Nealey, and J. J. de Pablo, Phys. Rev. Lett. 85, 3221 (2000).

10 T R. Böhme and J. J. de Pablo, J. Chem. Phys. 116, 9939 (2002).

11 P. G. de Gennes, Eur. Phys. J. E 2, 201 (2000); D. Long and F. Lequeux, Eur. Phys. J. E 4, 371 (2001).

${ }^{12}$ J. D. McCoy and J. G. Curro, J. Chem. Phys. 116, 9154 (2002).

${ }^{13}$ W. Kauzmann, Chem. Rev. 43, 219 (1948); J. H. Gibbs and E. A. Dimarzio, J. Chem. Phys. 28, 373 (1958); T. R. Kirkpatrick and P. G. Wolynes, Phys. Rev. B 36, 8552 (1987); F. H. Stillinger, J. Chem. Phys. 88, 7818 (1988); M. Mézard and G. Parisi, Phys. Rev. Lett. 82, 747 (1999).

${ }^{14}$ F. H. Stillinger and T. A. Weber, Phys. Rev. A 25, 978 (1982).

${ }^{15}$ P. G. Debenedetti, F. H. Stillinger, T. M. Truskett, and C. J. Roberts, J. Phys. Chem. B. 103 (1999); P. G. Debenedetti, T. M. Truskett, C. P. Lewis, and F. H. Stillinger, Adv. Chem. Eng. 28, 21 (2001); F. H. Stillinger, P. G. Debenedetti, and T. M. Truskett 105, 11809 (2001).

${ }^{16}$ M. S. Shell, P. G. Debenedetti, E. La Nave, and F. Sciortino, J. Chem. Phys 118, 8821 (2003).

${ }^{17}$ M. Schoen and D. J. Diestler, J. Chem. Phys. 109, 5596 (1998).

${ }^{18}$ T. M. Truskett, P. G. Debenedetti, and S. Torquato, J. Chem. Phys. 1142401 (2001). 
19 The mean-field expressions given in Eq. (4) represent the case where the effective "hardcore" diameters of the fluid-fluid and fluid-substrate interactions are equal.

${ }^{20}$ A. Scala, F. W. Starr, E. La Nave, F. Sciortino, and H. E. Stanley, Nature 406, 166 (2000); S. Sastry, Nature 409, 164 (2001); I. Saika-Voivod, P. H. Poole, and F. Sciortino, Nature 412, 514 (2001).

${ }^{21}$ R. Di Leonardo, L. Angelani, G. Parisi, and G. Ruocco, Phys. Rev. Lett. 84, 6054 (2000).

${ }^{22}$ T. M. Truskett and V. Ganesan, unpublished. 


\section{FIGURE CAPTIONS}

FIG. 1. The confinement induced shift in the IG transition $\Theta$ as a function of $\epsilon_{L}$ for the SSMF fluid film. The solid lines represent the approximation of Eq. (13) and the symbols are the predictions of the full nonlinear model of Eq. (11) and (12). The relative strength of the fluid-substrate to fluid-fluid attractions is quantified by $\Psi_{0} \sigma_{S S}^{3} / a_{b}$. The above results correspond to the case wherein the films are in equilibrium with the bulk SSMF fluid at $P_{b}=0$. 


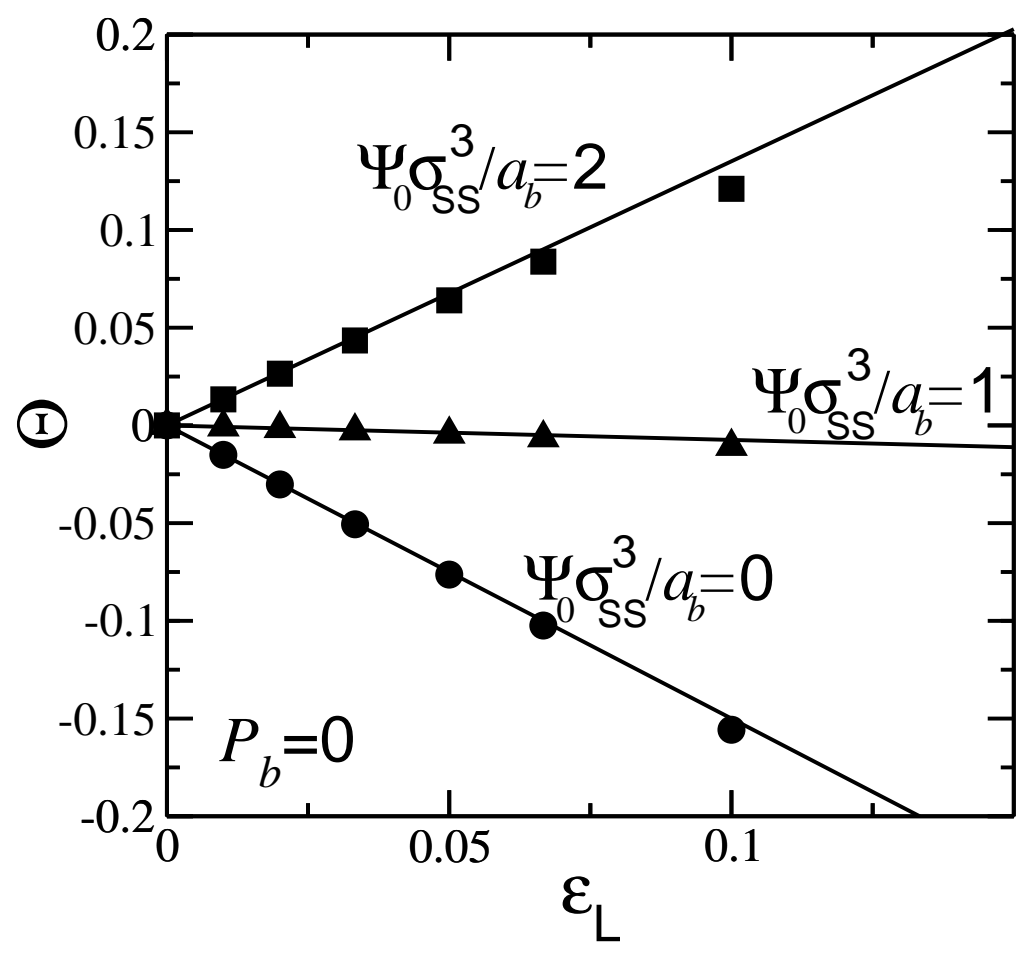

FIG. 1 - Truskett and Ganesan, 2003 